\title{
Static and Dynamic Analysis of the Closing Rotor Balancing Device of the Multistage Centrifugal Pump
}

\author{
Ivan Pavlenko ${ }^{1, a}$ \\ ${ }^{1}$ Sumy State University, Department of General Mechanics and Dynamics of Machines, \\ Rimskiy-Korsakov str. 2, 40007 Sumy, Ukraine \\ aivan_pavlenko@omdm.sumdu.edu.ua
}

\begin{abstract}
Keywords: centrifugal pump, axial force, face gap, pressure difference regulator, static characteristics, dynamic characteristics.

Abstract. In this paper the methods of static and dynamic analysis of the closing rotor balancing device of the multistage centrifugal pump are represented. On an example of the feed pump PE 600-300 static and dynamic characteristics are determined. Defined the resonance frequencies and investigated stability of the system "pump rotor - automatic balancing device - pressure difference regulator". The dependence for the pressure difference and conditions to ensure reliable operation of the locking rotor balancing device are determined.
\end{abstract}

\section{Introduction}

Axial rotor balancing in multistage centrifugal pumps is carried out mainly by using the unloading plungers. The residual axial force is unloaded by end bearings. Leakage of working fluid is limited by end seals. Such systems are the most often applied constructions, but they are complicated. They consist of piston, seal and axial bearing.

Paper [1] presents a new design of the axial forces balancing device of the multistage centrifugal machine, which has automatic unloading disc working in sealing liquid. That balancing device completely closes pump. Paper [2] presents the static characteristics of closing automatic rotor balancing device (CRBD) in the multistage centrifugal machine. These characteristics are obtained basing on equations of rotor axial equilibrium and flow balance. This device is complex liquiddynamic system with feedback that under certain conditions may have intensive self-oscillations, which affects the vibration state of the pump.

In papers [3-4] is represented methodology of static and dynamic calculations of the centrifugal machine rotor characteristics. Paper [5,6] considers linearised dynamic equations of simplified device design for laminar and turbulent gas flow regime.

The results of those articles require additional work which would take into account the real fluid flow regime, refinement of expressions for calculation leakages through throttling gaps. New design must include the regulator of pressure difference (RPD) to ensure the constant pressure difference between closing and working fluids.

\section{Description of the Locking Rotor Balancing Device}

CRBD consists of two subsystems: automatic rotor balancing device and RPD (Fig. 1). Axial force $\mathrm{T}$ acting on the rotor is balanced by unloading disc 1 . Closing fluid is supplied to the chamber on front of disc through the gap ge. Pressure in the chambers 3 and 6 depends on the face gap $\mathrm{z}$. Random change of axial force $T$ changes the value of face gap $z$. Thus, pressure difference $p_{2}-p_{3}$ takes the value that provides axial rotor balancing.

To prevent working fluid leakages it is necessary to provide overpressure of closing fluid. Thus, the device must have RPD. Inlet pressure $p_{\text {in }}>p_{1}$ is reduced to pressure $p_{\text {cam }}$ in the chamber 13 . Closing fluid with pressure $p_{e}$ in the chamber 14 under the membrane 9 is supplied to the chamber 3 . The chamber 15 over membrane is connected to the pump cave above the last impeller. 
Increasing of pressure $p_{1}$ increases the value of valve head gap 12 and pressure $p_{e}$. Thus, pressure difference $\delta p=p_{e}-p_{1}$ is unchanged.

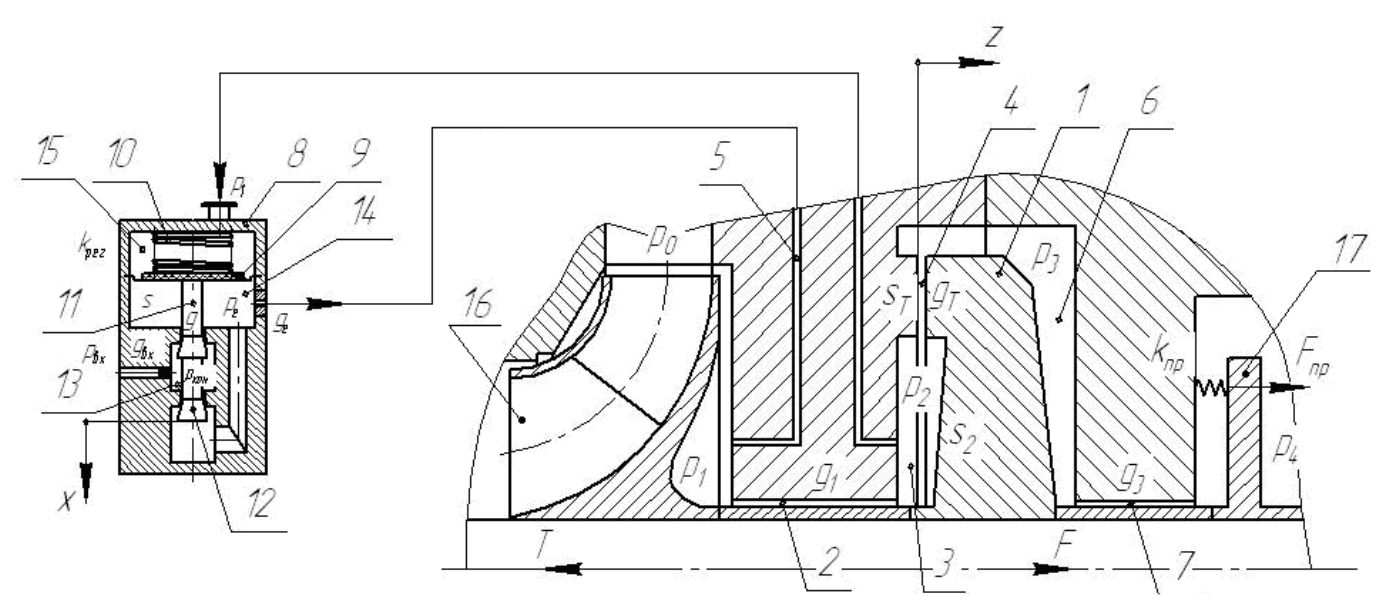

Fig. 1. CRBD scheme: 1 - unloading disc; 2 - cylindrical gap; 3 - chamber on front of disc; 4 - face gap; 5 - pulse channel; 6 - chamber behind of disc; 7 - outlet axial gap; 8 - RPD housing;

9 - membrane; 10, 17 - spring elements; 11 - rod; 12 - valve head; 13 - RPD inlet chamber; 14, 15 - membrane chambers; 16 - last stage impeller

\section{Static Analysis}

Static calculation of CRBD allows at design stage to choose the basic geometric parameters of the hydro-mechanical system provided that in a given range of axial force changing the values of face gap and leakages did not exceed the permissible limits. Static calculation is based on a compatible solution of the equations of axial equilibrium of the pump rotor and RPD rod and balance equations through throttling gaps according to the hydraulic path (Fig. 2).

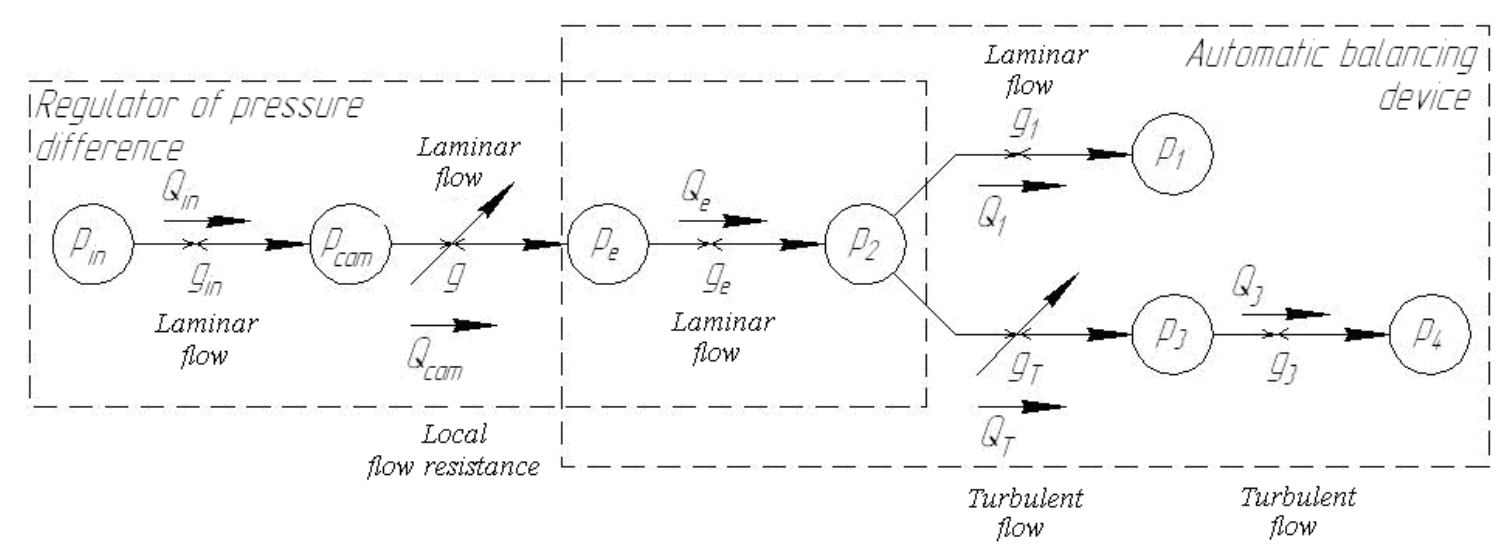

Fig. 2. Hydraulic path scheme: $Q_{\text {in }}$ - inlet leakage; $Q_{\text {cam }}$ - leakage through throttling gap of RPD; $\mathrm{Q}_{\mathrm{e}}$ - closing fluid leakage; $\mathrm{Q}_{1}, \mathrm{Q}_{3}, \mathrm{Q}_{\mathrm{T}}$ - leakages through inlet, outlet and face gaps

The equation of axial rotor equilibrium $\mathrm{T}=\mathrm{F}+\mathrm{F}_{\mathrm{spr}}$ contains force of previous deformation of the spring element 17 (Fig. 1$) \mathrm{F}_{\mathrm{spr}}=\mathrm{k}_{\mathrm{spr}}\left(\Delta_{\mathrm{spr}}-\mathrm{z}\right.$ ), where $\mathrm{k}_{\mathrm{spr}}, \Delta_{\text {spr }}$ are stiffness coefficient and previous deformations of the spring element. Provided that $\mathrm{z}<<\Delta_{\text {spr }}$ force $\mathrm{F}_{\mathrm{spr}}=\mathrm{k}_{\mathrm{spr}} \Delta_{\text {spr }}$. Unloading force $\mathrm{F}=$ $\mathrm{p}_{2} \mathrm{~s}_{2}+0.5\left(\mathrm{p}_{2}+\mathrm{p}_{3}\right) \mathrm{s}_{\mathrm{T}}-\mathrm{p}_{3}\left(\mathrm{~s}_{\mathrm{T}}+\mathrm{s}_{\mathrm{T}}\right)=\mathrm{s}_{\mathrm{e}}\left(\mathrm{p}_{2}-\mathrm{p}_{3}\right)$ is is proportional to the effective area $\mathrm{s}_{\mathrm{e}}=\mathrm{s}_{2}+0.5 \mathrm{~s}_{\mathrm{T}}$, depending on the area $\mathrm{s}_{2}$ under the face gap and area $\mathrm{s}_{\mathrm{T}}$ of the face gap. Thus, the equation of axial rotor equilibrium is $\mathrm{s}_{\mathrm{e}}\left(\mathrm{p}_{2}-\mathrm{p}_{3}\right)=\mathrm{T}-\mathrm{k}_{\mathrm{spr}} \Delta_{\mathrm{spr}}$.

The equation of axial RPD rod equilibrium $s_{m}\left(p_{e}-p_{1}\right)=F_{\text {reg }}$ contains the membrane area $s_{m}$ and control RPD action $\mathrm{F}_{\text {reg }}=\mathrm{k}_{\mathrm{reg}}\left(\Delta_{\text {reg }}-\mathrm{x}\right)$, where $\mathrm{k}_{\text {reg }}, \Delta_{\text {reg }}$ are stiffness coefficient and previous deformations of the spring element 10 (Fig. 1), $\mathrm{x}$ - the value of throttling gap of RPD. Provided that $\mathrm{x}<<\Delta_{\text {reg }}$ force $\mathrm{F}_{\text {reg }}=\mathrm{k}_{\text {reg }} \Delta_{\text {reg. }}$. 
Leakages in balance equations $\mathrm{Q}_{\text {in }}=\mathrm{Q}_{\text {cam }}=\mathrm{Q}_{\mathrm{e}}=\mathrm{Q}_{1}+\mathrm{Q}_{\mathrm{T}}$ and $\mathrm{Q}_{\mathrm{T}}=\mathrm{Q}_{3}$ depend on the fluid flow regime. Pressure difference on the inlet and outlet RPD throttles and through inlet axial throttle has the order $1 \mathrm{MPa}$, and Reynolds number has the order $10^{3}$, therefore the flow regime is laminar and leakages $\mathrm{Q}_{\text {in }}=\mathrm{g}_{\text {in }}\left(\mathrm{p}_{\mathrm{in}}-\mathrm{p}_{\text {cam }}\right), \mathrm{Q}_{\mathrm{e}}=\mathrm{g}_{\mathrm{e}}\left(\mathrm{p}_{\mathrm{e}}-\mathrm{p}_{2}\right), \mathrm{Q}_{1}=\mathrm{g}_{1}\left(\mathrm{p}_{2}-\mathrm{p}_{1}\right)$ are proportional to pressure differences, where $g_{i n}, g_{e}, g_{1}$ are conductivity coefficients. Pressure difference on the face gap and outlet axial throttle has the order $10 \mathrm{MPa}$, Reynolds number $-10^{5}$, therefore the flow regime is turbulent and leakages $\mathrm{Q}_{\mathrm{T}}=\mathrm{g}_{\mathrm{tb}} \mathrm{u}^{3 / 2}\left(\mathrm{p}_{2}-\mathrm{p}_{3}\right)^{1 / 2}, \mathrm{Q}_{3}=\mathrm{g}_{3}\left(\mathrm{p}_{3}-\mathrm{p}_{4}\right)^{1 / 2}$, where $\mathrm{u}=\mathrm{z} / \mathrm{z}_{\mathrm{b}}$ is the the dimensionless value of face gap $\left(\mathrm{z}_{\mathrm{b}}\right.$ is the base gap value); $\mathrm{g}_{\mathrm{tb}}, \mathrm{g}_{3}$ are gaps conductivities. Leakage through the RPD throttle $\mathrm{Q}_{\mathrm{cam}}=\mathrm{g}_{\mathrm{b}} \xi\left(\mathrm{p}_{\mathrm{cam}}-\mathrm{p}_{\mathrm{e}}\right)^{1 / 2}$ depends on the dimensionless value $\xi=\mathrm{x} / \mathrm{x}_{\mathrm{b}}$ of throttling gap and conductivity coefficient $\mathrm{g}_{\mathrm{b}}\left(\mathrm{x}_{\mathrm{b}}\right.$ is the dimensionless value of throttling gap).

Finally the equations of static calculation of CRBD are

$$
\begin{aligned}
& \mathrm{s}_{\mathrm{e}}\left(\mathrm{p}_{2}-\mathrm{p}_{3}\right)=\mathrm{T}-\mathrm{k}_{\mathrm{spr}} \Delta_{\mathrm{spr}} ; \mathrm{s}_{\mathrm{m}}\left(\mathrm{p}_{\mathrm{e}}-\mathrm{p}_{1}\right)=\mathrm{k}_{\mathrm{reg}} \Delta_{\mathrm{reg}} ; \\
& \mathrm{g}_{\mathrm{in}}\left(\mathrm{p}_{\mathrm{in}}-\mathrm{p}_{\mathrm{cam}}\right)=\mathrm{g}_{\mathrm{b}} \xi\left(\mathrm{p}_{\mathrm{cam}}-\mathrm{p}_{\mathrm{e}}\right)^{1 / 2} ; \mathrm{g}_{\mathrm{b}} \xi\left(\mathrm{p}_{\mathrm{cam}}-\mathrm{p}_{\mathrm{e}}\right)^{1 / 2}=\mathrm{g}_{\mathrm{e}}\left(\mathrm{p}_{\mathrm{e}}-\mathrm{p}_{2}\right) ; \\
& \mathrm{g}_{\mathrm{e}}\left(\mathrm{p}_{\mathrm{e}}-\mathrm{p}_{2}\right)=\mathrm{g}_{1}\left(\mathrm{p}_{2}-\mathrm{p}_{1}\right)+\mathrm{g}_{\mathrm{tb}} \mathrm{u}^{3 / 2}\left(\mathrm{p}_{2}-\mathrm{p}_{3}\right)^{1 / 2} ; \mathrm{g}_{\mathrm{tb}} \mathrm{u}^{3 / 2}\left(\mathrm{p}_{2}-\mathrm{p}_{3}\right)^{1 / 2}=\mathrm{g}_{3}\left(\mathrm{p}_{3}-\mathrm{p}_{4}\right)^{1 / 2}
\end{aligned}
$$

For further calculations are used dimensionless parameters: pressures $\psi_{\text {in,cam,e, }, 2,3,4}=$ $=p_{\text {in,cam,e, } 1,2,3,4} / p_{b}$, where $p_{b}$ is base (nominal discharge) pressure; axial force $\tau=T /\left(p_{b} S_{b}\right)=b \psi_{1}$, where $s_{b}$ is the base (inlet impeller) area, $b=1-\rho \omega^{2} R_{2}^{2} j\left[1-\left(R_{0}^{2}+R_{1}^{2}\right) /\left(2 R_{2}^{2}\right)\right] /\left(8 p_{b}\right)-$ proportional coefficient ( $\rho$ - fluid density, $\omega$ - angular ( $i$ is imaginary unit, $\omega$ is angular frequency of the rotor, $\mathrm{R}_{0,1}$ - inner radiuses of the primary impeller disc, $\mathrm{R}_{2}$ - external impeller radius, $\mathrm{g}-$ free fall acceleration, $\mathrm{j}$ - number of the pump stages); spring forces $\chi=\mathrm{k}_{\mathrm{spr}} \Delta_{\mathrm{spr}} /\left(\mathrm{p}_{\mathrm{b}} \mathrm{s}_{\mathrm{b}}\right)$, $\chi_{\text {reg }}=\mathrm{k}_{\mathrm{reg}} \Delta_{\text {reg }} /\left(\mathrm{p}_{\mathrm{b}} \mathrm{s}_{\mathrm{b}}\right)$; effective and membrane areas $\sigma=\mathrm{s}_{\mathrm{e}} / \mathrm{s}_{\mathrm{b}}, \sigma_{\mathrm{m}}=\mathrm{s}_{\mathrm{m}} / \mathrm{s}_{\mathrm{b}}$; conductivity ratio $\alpha_{\mathrm{in} 1, \mathrm{el}}=\mathrm{g}_{\mathrm{in}, \mathrm{e}} / \mathrm{g}_{1}, \alpha_{\mathrm{b} 1, \mathrm{tb} 1,31}=\mathrm{g}_{\mathrm{b} 1, \mathrm{tb} 1,31} /\left(\mathrm{g}_{1} \mathrm{p}_{\mathrm{b}}{ }^{1 / 2}\right)$. Thus, system of Eq. 1 takes the form

$$
\begin{aligned}
& \sigma\left(\psi_{2}-\psi_{3}\right)=\mathrm{b} \psi_{1}-\chi ; \delta \psi=\psi_{\mathrm{e}}-\psi_{1}=\chi_{\mathrm{reg}} / \sigma_{\mathrm{m}} ; \\
& \alpha_{\mathrm{in} 1}{ }^{2}\left(\psi_{\mathrm{in}}-\psi_{\mathrm{cam}}\right)^{2}=\alpha_{\mathrm{b} 1} \xi^{2}\left(\psi_{\mathrm{cam}}-\psi_{\mathrm{e}}\right) ; \alpha_{\mathrm{in} 1}\left(\psi_{\mathrm{in}}-\psi_{\mathrm{cam}}\right)=\alpha_{\mathrm{el}}\left(\psi_{\mathrm{e}}-\psi_{2}\right) ; \\
& \alpha_{\mathrm{tb} 1} \mathrm{u}^{3}\left(\psi_{2}-\psi_{3}\right)=\left[\alpha_{\mathrm{e} 1}\left(\psi_{1}+\delta \psi-\psi_{2}\right)-\psi_{2}+\psi_{1}\right]^{2} ; \alpha_{\mathrm{tb} 1} \mathrm{u}^{3}\left(\psi_{2}-\psi_{3}\right)=\alpha_{31}{ }^{2}\left(\psi_{3}-\psi_{4}\right),
\end{aligned}
$$

where $\delta \psi$ is dimensionless pressure difference as the main parameter of static calculation of CRBD.

Static analysis of CRBD reduces to determining pressures on CRBD chambers, face gap value and leakages through throttles as functions of the discharge pressure $\psi_{1}$.

Under the conditions $\delta \psi>>0.5\left(\alpha_{31} / \alpha_{\mathrm{e} 1}\right)^{2}, \psi_{1}>>\mathrm{b}\left(\alpha_{31} / \alpha_{\mathrm{e} 1}\right)^{2} / \sigma, \psi_{4} \approx 0, \chi<<\tau$ from Eq. 2

$$
\begin{aligned}
& \psi_{2}=\psi_{1}+\delta \psi \cdot \alpha_{\mathrm{e} 1} /\left(1+\alpha_{\mathrm{e} 1}\right) ; \psi_{3}=(1-\mathrm{b} / \sigma) \psi_{1}+\delta \psi \cdot \alpha_{\mathrm{e} 1} /\left(1+\alpha_{\mathrm{e} 1}\right)+\chi / \sigma ; \\
& \mathrm{u}=\left\{\left(\alpha_{\mathrm{e} 1} / \alpha_{\mathrm{tb} 1}\right)^{2}\left[(1-\mathrm{b} / \sigma) \psi_{1}+\delta \psi \cdot \alpha_{\mathrm{e} 1} /\left(1+\alpha_{\mathrm{e} 1}\right)+\chi / \sigma-\psi_{4}\right] /\left(\mathrm{b} \psi_{1}-\chi\right)\right\}^{1 / 3} \\
& \psi_{\mathrm{cam}}=\psi_{\mathrm{in}}-\delta \psi \cdot \alpha_{\mathrm{e} 1} /\left[\alpha_{\mathrm{in} 1}\left(1+\alpha_{\mathrm{e} 1}\right)\right] ; \psi_{\mathrm{e}}=\psi_{1}+\delta \psi ; \\
& \xi=\delta \psi \cdot \alpha_{\mathrm{e} 1} /\left[\alpha_{\mathrm{b} 1}\left(1+\alpha_{\mathrm{e} 1}\right)\right] /\left\{\psi_{\mathrm{in}}-\psi_{1}-\delta \psi \cdot\left[\alpha_{\mathrm{in} 1}+\alpha_{\mathrm{e} 1}\left(1+\alpha_{\mathrm{in} 1}\right)\right] /\left[\alpha_{\mathrm{in} 1}\left(1+\alpha_{\mathrm{e} 1}\right)\right]\right\}^{1 / 2}
\end{aligned}
$$

Dimensionless leakages through throttles are determined by the formulas

$$
\begin{aligned}
& \mathrm{q}_{\mathrm{T}}=\mathrm{Q}_{\mathrm{T}} / \mathrm{Q}_{\mathrm{e}}=\alpha_{31}\left(1+\alpha_{\mathrm{e} 1}\right) /\left(\alpha_{\mathrm{e}} \delta \psi\right)\left[(1-\mathrm{b} / \sigma) \psi_{1}+\delta \psi \cdot \alpha_{\mathrm{e} 1} /\left(1+\alpha_{\mathrm{e} 1}\right)+\chi / \sigma\right]^{1 / 2} ; \mathrm{q}_{\mathrm{e}}=1 \\
& \mathrm{q}_{1}=\mathrm{Q}_{1} / \mathrm{Q}_{\mathrm{e}}=1-\mathrm{q}_{\mathrm{T}}=1-\alpha_{31}\left(1+\alpha_{\mathrm{e} 1}\right) /\left(\alpha_{\mathrm{e} 1} \delta \psi\right)\left[(1-\mathrm{b} / \sigma) \psi_{1}+\delta \psi \cdot \alpha_{\mathrm{e} 1} /\left(1+\alpha_{\mathrm{e} 1}\right)+\chi / \sigma\right]^{1 / 2}
\end{aligned}
$$

where $\mathrm{Q}_{\mathrm{e}}=\mathrm{g}_{\mathrm{e}} \mathrm{p}_{\mathrm{b}} \delta \psi /\left(1+\alpha_{\mathrm{e} 1}\right)$ is constant leakage of closing fluid.

Reliable operation of the CRBD is only possible in the absence of leakage of the working fluid to the chamber on front of face gap $\left(q_{1} \geq 0\right)$. Under this condition from Eq. 3 the minimum pressure difference $\delta \psi_{\min }=\alpha_{31}\left(1+\alpha_{\mathrm{e} 1}\right) / \alpha_{\mathrm{e} 1}[1-(b-\chi) / \sigma]^{1 / 2}$. The working fluid leaks into the chamber on front of the face throttle in excess of the critical value of the dimensionless discharge pressure $\psi_{1}{ }^{\mathrm{cr}}=\left\{\left[\alpha_{\mathrm{e} 1} / \alpha_{31} \cdot \delta \psi /\left(1+\alpha_{\mathrm{e} 1}\right)\right]^{2}-\delta \psi \cdot \alpha_{\mathrm{e} 1} /\left(1+\alpha_{\mathrm{e} 1}\right)+\chi / \sigma\right\} /(1-\mathrm{b} / \sigma)$.

Static characteristics of CRBD as dependence of the dimensionless unloading force $\varphi=\sigma\left(\psi_{2}-\psi_{3}\right)=\sigma\left[\psi_{1}-\psi_{4}+\delta \psi \cdot \alpha_{\mathrm{el}} /\left(1+\alpha_{\mathrm{e} 1}\right)\right] /\left[1+\left(\alpha_{\mathrm{tb} 1} / \alpha_{31}\right)^{2} \mathrm{u}^{3}\right]$ on the face gap $\mathrm{u}$ allows to 
determine the hydro-static stiffness coefficient $\kappa_{\mathrm{s}}=\partial \varphi / \partial \mathrm{u}=-3 \sigma / \mathrm{u}\left[\psi_{1}-\psi_{4}+\delta \psi \cdot \alpha_{\mathrm{e} 1} /\left(1+\alpha_{\mathrm{e} 1}\right)\right] \times$ $\times\left(\alpha_{\mathrm{tb} 1} / \alpha_{31}\right)^{2} \mathrm{u}^{3} /\left[1+\left(\alpha_{\mathrm{tb} 1} / \alpha_{31}\right)^{2} \mathrm{u}^{3}\right]$, which is always negative and indicates the static stability of CRBD.

\section{Dynamic Analysis}

Dynamic analysis of the CRBD is to determine the dynamic characteristics based on the equations of axial movement of the pump rotor $\left(\mathrm{m}_{\mathrm{r}} \mathrm{d}^{2} \mathrm{z} / \mathrm{dt}^{2}+\mathrm{c}_{\mathrm{z}} \mathrm{dz} / \mathrm{dt}+\mathrm{k}_{\mathrm{spr}} \mathrm{z}=\mathrm{F}-\mathrm{T}+\mathrm{F}_{\mathrm{spr}}\right)$ and the RPD rod $\left(m_{0} d^{2} x / d t^{2}+c_{x} d z / d t+k_{\text {reg }} x=F_{\text {reg }}-F_{m}\right)$ and balance equations through throttling gaps (Fig. 2): $\mathrm{Q}_{\text {in }}=\mathrm{Q}_{\text {cam }}+\mathrm{Q}_{\text {cam }}{ }^{\mathrm{p}}+\mathrm{Q}_{\text {cam }}{ }^{\mathrm{v}}=\mathrm{Q}_{\mathrm{e}}+\mathrm{Q}_{\mathrm{e}}^{\mathrm{p}}+\mathrm{Q}_{\mathrm{e}}^{\mathrm{v}}=\mathrm{Q}_{1}+\mathrm{Q}_{\mathrm{T}}+\mathrm{Q}_{\mathrm{T}}{ }^{\mathrm{p}}+\mathrm{Q}_{\mathrm{T}}{ }^{\mathrm{v}} ; \mathrm{Q}_{\mathrm{T}}+\mathrm{Q}_{\mathrm{T}}{ }^{\mathrm{p}}+\mathrm{Q}_{\mathrm{T}}{ }^{\mathrm{v}}=$ $=\mathrm{Q}_{3}+\mathrm{Q}_{3}{ }^{\mathrm{p}}+\mathrm{Q}_{3}{ }^{\mathrm{v}}$, where $\mathrm{z}, \mathrm{x}-$ axial movement of the pump rotor and $\mathrm{RPD} \operatorname{rod} ; \mathrm{m}_{\mathrm{r}}, \mathrm{m}_{0}-$ rotor and rod masses; $\mathrm{c}_{\mathrm{z}}, \mathrm{c}_{\mathrm{x}}-$ damping coefficients; $\mathrm{Q}_{\text {cam }}{ }^{\mathrm{p}}, \mathrm{Q}_{\mathrm{e}}{ }^{\mathrm{p}}, \mathrm{Q}_{\mathrm{T}}{ }^{\mathrm{p}}, \mathrm{Q}_{3}{ }^{\mathrm{p}}, \mathrm{Q}_{\mathrm{cam}}{ }^{\mathrm{v}}, \mathrm{Q}_{\mathrm{e}}{ }^{\mathrm{v}}, \mathrm{Q}_{\mathrm{T}}{ }^{\mathrm{v}}, \mathrm{Q}_{3}{ }^{\mathrm{v}}-$ compressing and displacement leakages: $\mathrm{Q}_{\text {cam }}{ }^{\mathrm{p}}=\mathrm{V}_{\text {cam }} / \mathrm{E} \cdot \mathrm{dp}_{\mathrm{cam}} / \mathrm{dt}, \mathrm{Q}_{\mathrm{e}}^{\mathrm{p}}=\mathrm{V}_{\mathrm{m}} / \mathrm{E} \cdot \mathrm{dp}_{\mathrm{e}} / \mathrm{dt}, \mathrm{Q}_{\mathrm{T}}{ }^{\mathrm{p}}=\mathrm{V}_{2} / \mathrm{E} \cdot \mathrm{dp}_{2} / \mathrm{dt}$, $\mathrm{Q}_{3}{ }^{\mathrm{p}}=\mathrm{V}_{3} / \mathrm{E} \cdot \mathrm{dp}_{3} / \mathrm{dt}, \mathrm{Q}_{\mathrm{cam}}{ }^{\mathrm{v}}=\mathrm{s}_{\mathrm{c}} \mathrm{dx} / \mathrm{dt}, \mathrm{Q}_{\mathrm{e}}{ }^{\mathrm{v}}=\mathrm{s}_{\mathrm{m}} \mathrm{dx} / \mathrm{dt}, \mathrm{Q}_{\mathrm{T}}{ }^{\mathrm{v}}=\mathrm{Q}_{3}{ }^{\mathrm{v}}=\mathrm{se}_{\mathrm{e}} \mathrm{dz} / \mathrm{dt} ; \mathrm{V}_{\text {cam }}, \mathrm{V}_{\mathrm{m}}, \mathrm{V}_{2}, \mathrm{~V}_{3}-$ volumes of hydraulic path chambers; $s_{c}$ - contact area of the RPD saddle; $E$ - modulus of elasticity of closing fluid. Finally, the system of equations for dynamic analysis:

$$
\begin{aligned}
& \mathrm{m}_{\mathrm{r}} \mathrm{d}^{2} \mathrm{z} / \mathrm{dt}^{2}+\mathrm{c}_{\mathrm{z}} \mathrm{dz} / \mathrm{dt}+\mathrm{k}_{\mathrm{spr}} \mathrm{z}=\mathrm{F}-\mathrm{T}+\mathrm{F}_{\mathrm{spr}} ; \mathrm{m}_{0} \mathrm{~d}^{2} \mathrm{x} / \mathrm{dt}^{2}+\mathrm{c}_{\mathrm{x}} \mathrm{dz} / \mathrm{dt}+\mathrm{k}_{\mathrm{reg}} \mathrm{x}=\mathrm{F}_{\mathrm{reg}}-\mathrm{F}_{\mathrm{m}} ; \\
& \mathrm{g}_{\mathrm{in}}\left(\mathrm{p}_{\mathrm{in}}-\mathrm{p}_{\mathrm{cam}}\right)=\mathrm{g}_{\mathrm{b}} \xi\left(\mathrm{p}_{\mathrm{cam}}-\mathrm{p}_{\mathrm{e}}\right)^{1 / 2}+\mathrm{V}_{\mathrm{cam}} / \mathrm{E} \cdot \mathrm{dp}_{\mathrm{cam}} / \mathrm{dt}+\mathrm{s}_{\mathrm{c}} \mathrm{dx} / \mathrm{dt}=\mathrm{ge}_{\mathrm{e}}\left(\mathrm{p}_{\mathrm{e}}-\mathrm{p}_{2}\right)+\mathrm{V}_{\mathrm{m}} / \mathrm{E} \cdot \mathrm{dp} \mathrm{e} / \mathrm{dt}+ \\
& +\mathrm{s}_{\mathrm{m}} \mathrm{dx} / \mathrm{dt}=\mathrm{g}_{1}\left(\mathrm{p}_{2}-\mathrm{p}_{1}\right)+\mathrm{g}_{\mathrm{tb}} \mathrm{u}^{3 / 2}\left(\mathrm{p}_{2}-\mathrm{p}_{3}\right)^{1 / 2}+\mathrm{V}_{2} / \mathrm{E} \cdot \mathrm{dp}_{2} / \mathrm{dt}+\mathrm{s}_{\mathrm{e}} \mathrm{dz} / \mathrm{dt} \\
& \mathrm{g}_{\mathrm{tb}} \mathrm{u}^{3 / 2}\left(\mathrm{p}_{2}-\mathrm{p}_{3}\right)^{1 / 2}+\mathrm{V}_{2} / \mathrm{E} \cdot \mathrm{dp}_{2} / \mathrm{dt}+\mathrm{s}_{\mathrm{e}} \mathrm{dz} / \mathrm{dt}=\mathrm{g}_{3}\left(\mathrm{p}_{3}-\mathrm{p}_{4}\right)^{1 / 2}+\mathrm{V}_{3} / \mathrm{E} \cdot \mathrm{dp}_{3} / \mathrm{dt}+\mathrm{s}_{\mathrm{e}} \mathrm{dz} / \mathrm{dt} .
\end{aligned}
$$

The system of nonlinear differential equations (5) can not be solved analytically. Further research is conducted for variations of time-variables parameters (" $\delta$ " is variation sign) by linearization $\mathrm{z}=\mathrm{z}_{0}$ $+\delta \mathrm{z}, \mathrm{u}=\mathrm{u}_{0}+\delta \mathrm{u}, \mathrm{x}=\mathrm{x}_{0}+\delta \mathrm{x}, \xi=\xi_{0}+\delta \xi, \mathrm{T}=\mathrm{T}_{0}+\delta \mathrm{T}, \mathrm{F}=\mathrm{F}_{0}+\delta \mathrm{F}$, $\mathrm{p}_{1}=\mathrm{p}_{10}+\delta \mathrm{p}_{1}, \mathrm{p}_{\mathrm{cam}}=\mathrm{p}_{\mathrm{cam} 0}+\delta \mathrm{p}_{\mathrm{cam}}, \mathrm{p}_{\mathrm{e}}=\mathrm{p}_{\mathrm{e} 0}+\delta \mathrm{p}_{\mathrm{e}}, \mathrm{p}_{2}=\mathrm{p}_{20}+\delta \mathrm{p}_{2}, \mathrm{p}_{3}=\mathrm{p}_{30}+\delta \mathrm{p}_{3}$ relatively to stationary values (with index " 0 ") as a result (2) of solving the system of algebraic equations (1).

For further calculations are used dimensionless parameters: $\delta \psi_{\text {cam }}=\delta \mathrm{p}_{\text {cam }} / \mathrm{p}_{\mathrm{b}}, \delta \psi_{\mathrm{e}}=\delta \mathrm{p}_{\mathrm{e}} / \mathrm{p}_{\mathrm{b}}$, $\delta \psi_{1}=\delta \mathrm{p}_{1} / \mathrm{p}_{\mathrm{b}}, \delta \psi_{2}=\delta \mathrm{p}_{2} / \mathrm{p}_{\mathrm{b}}, \delta \psi_{3}=\delta \mathrm{p}_{3} / \mathrm{p}_{\mathrm{b}}, \delta \tau=\delta \mathrm{T} /\left(\mathrm{p}_{\mathrm{b}} \mathrm{s}_{\mathrm{b}}\right)=\mathrm{b} \delta \psi_{1}, \delta \varphi=\delta \mathrm{F} /\left(\mathrm{p}_{\mathrm{b}} \mathrm{s}_{\mathrm{b}}\right)$. System of dynamics equations (5) can be represented in matrix and operator form $\mathrm{N} \delta \mathrm{U}=\mathrm{B} \delta \psi_{1}$, where $\mathrm{N}$ is the matrix of differentiation operators, $\delta \mathrm{U}-$ is reaction of the system to the external action $\mathrm{B} \delta \psi_{1}$ :

$$
\begin{aligned}
& \mathrm{N}=\left\{\mathrm{N}_{1}, \mathrm{~N}_{2}, \mathrm{~N}_{3}, \mathrm{~N}_{4}, \mathrm{~N}_{5}, \mathrm{~N}_{6}\right\}^{\mathrm{T}} ; \mathrm{N}_{1}=\left\{\mathrm{K}_{1}\left(\mathrm{~T}_{1}^{2}+2 \zeta_{1} \mathrm{~T}_{1} \mathrm{p}+1\right), 0,0,0,-\sigma, \sigma\right\}, \\
& \mathrm{N}_{2}=\left\{0, \mathrm{~K}_{2}\left(\mathrm{~T}_{2}^{2}+2 \zeta_{2} \mathrm{~T}_{2} \mathrm{p}+1\right), 0, \sigma_{\mathrm{m}}, 0,0\right\}, \mathrm{N}_{3}=\left\{0, \mathrm{~K}_{4}\left(\mathrm{~T}_{4} \mathrm{p}+1\right), \mathrm{T}_{3} \mathrm{p}+1,-\mathrm{K}_{3}, 0,0\right\}, \\
& \mathrm{N}_{4}=\left\{0,-\mathrm{K}_{6}\left(\mathrm{~T}_{6} \mathrm{p}+1\right),-\mathrm{K}_{5}\left(\tau_{3} \mathrm{p}+1\right), \mathrm{T}_{5} \mathrm{p}+1,-\mathrm{K}_{7}, 0\right\}, \\
& \mathrm{N}_{5}=\left\{\mathrm{K}_{8}\left(\mathrm{~T}_{8} \mathrm{p}+1\right), \tau_{6} \mathrm{p}, 0,-\mathrm{K}_{9}\left(\tau_{5} \mathrm{p}+1\right), \mathrm{T}_{7} \mathrm{p}+1,-\mathrm{K}_{10}\right\}, \\
& \mathrm{N}_{6}=\left\{-\mathrm{K}_{12}, 0,0,0,-\mathrm{K}_{13}\left(\tau_{7} \mathrm{p}+1\right), \mathrm{T}_{9} \mathrm{p}+1\right\} ; \\
& \delta \mathrm{U}=\left\{\delta \mathrm{u} \delta \xi \delta \psi_{\text {cam }} \delta \psi_{\mathrm{e}} \delta \psi_{2} \delta \psi_{3}\right\}^{\mathrm{T}} ; \mathrm{B}=\left\{-\mathrm{b}, \sigma_{\mathrm{m}}, 0,0, \mathrm{~K}_{11}, 0\right\} .
\end{aligned}
$$

The matrix $\mathrm{N}$ contains 28 constant parameters: time constants $\mathrm{T}_{1 \ldots 9}$ and $\tau_{3,5,6,7}$, damping coefficients $\zeta_{1,2}$ and amplification factors $\mathrm{K}_{1 \ldots 13}$ :

$$
\begin{aligned}
& \mathrm{T}_{1}=\left(\mathrm{m}_{\mathrm{r}} / \mathrm{k}_{\mathrm{spr}}\right)^{1 / 2}, \mathrm{~T}_{2}=\left(\mathrm{m}_{0} / \mathrm{k}_{\mathrm{reg}}\right)^{1 / 2}, \mathrm{~T}_{3}=\mathrm{V}_{\mathrm{cam}} /\left(\mathrm{Eg}_{1}\right) /\left[\alpha_{\mathrm{in} 1}+0.5 \alpha_{\mathrm{b} 1} \xi_{0} /\left(\psi_{\mathrm{cam} 0}-\psi_{\mathrm{e} 0}\right)^{1 / 2}\right], \\
& \mathrm{T}_{4}=\mathrm{s}_{\mathrm{c}} \mathrm{Z}_{\mathrm{b}} /\left[\mathrm{g}_{1} \mathrm{p}_{\mathrm{b}} \alpha_{\mathrm{b} 1}\left(\psi_{\mathrm{cam} 0}-\psi_{\mathrm{e} 0}\right)^{1 / 2}\right], \mathrm{T}_{5}=\mathrm{V}_{\mathrm{m}} /\left(\mathrm{Eg}_{1}\right) /\left[0.5 \alpha_{\mathrm{b} 1} \xi_{0} /\left(\psi_{\mathrm{cam} 0}-\psi_{\mathrm{e} 0}\right)^{1 / 2}+\alpha_{\mathrm{e} 1}\right], \\
& \mathrm{T}_{6}=\left(\mathrm{s}_{\mathrm{c}}-\mathrm{s}_{\mathrm{m}}\right) \mathrm{z}_{\mathrm{b}} /\left(\mathrm{g}_{1} \alpha_{\mathrm{b} 1}\right) /\left[\mathrm{p}_{\mathrm{b}}\left(\psi_{\mathrm{cam} 0}-\psi_{\mathrm{e} 0}\right)\right]^{1 / 2} \\
& \mathrm{~T}_{7}=\mathrm{V}_{2} /\left(\mathrm{Eg}_{1}\right) /\left[1+\alpha_{\mathrm{e} 1}+0.5 \alpha_{\mathrm{tb} 1} \mathrm{u}_{0}^{3 / 2} /\left(\psi_{20}-\psi_{30}\right)^{1 / 2}\right] \\
& \mathrm{T}_{8}=2 \mathrm{~s}_{\mathrm{e}} \mathrm{Z}_{\mathrm{b}} /\left\{3 \mathrm{p}_{\mathrm{b}} \mathrm{g}_{1} \alpha_{\mathrm{tb} 1}\left[\mathrm{u}_{0}\left(\psi_{20}-\psi_{30}\right)\right]^{1 / 2}\right\} \\
& \mathrm{T}_{9}=2 \mathrm{~V}_{3} /\left(\mathrm{Eg}_{1}\right) /\left[\alpha_{\mathrm{tb} 1} \mathrm{u}_{0}^{3 / 2} /\left(\psi_{20}-\psi_{30}\right)^{1 / 2}+\alpha_{31} /\left(\psi_{30}-\psi_{4}\right)^{1 / 2}\right] \\
& \tau_{3}=2 \mathrm{~V}_{\mathrm{cam}}\left(\psi_{\mathrm{cam} 0}-\psi_{\mathrm{e} 0}\right)^{1 / 2} /\left(\mathrm{Eg}_{1} \alpha_{\mathrm{b} 1} \xi_{0}\right), \tau_{5}=\mathrm{V}_{\mathrm{m}} /\left(\mathrm{Eg}_{\mathrm{e}}\right), \\
& \tau_{6}=\mathrm{s}_{\mathrm{m}} \mathrm{Z}_{\mathrm{b}} /\left(\mathrm{p}_{\mathrm{b}} \mathrm{g}_{1}\right) /\left[1+\alpha_{\mathrm{e} 1}+0.5 \mathrm{\alpha}_{\mathrm{tb} 1} \mathrm{u}_{0}^{3 / 2} /\left(\psi_{20}-\psi_{30}\right)^{1 / 2}\right], \tau_{7}=2 \mathrm{~V}_{2}\left(\psi_{20}-\psi_{30}\right)^{1 / 2} /\left(\mathrm{Eg}_{1} \alpha_{\mathrm{tb} 1} \mathrm{u}_{0}{ }^{3 / 2}\right) \\
& \zeta_{1}=\mathrm{c}_{\mathrm{z}} /\left(2 \mathrm{k}_{\mathrm{spr}} \mathrm{T}_{1}\right), \zeta_{2}=\mathrm{c}_{\mathrm{x}} /\left(2 \mathrm{k}_{\mathrm{reg}} \mathrm{T}_{2}\right) ; \\
& \mathrm{K}_{1}=\chi, \mathrm{K}_{2}=\sigma_{\mathrm{m}} \delta \psi, \mathrm{K}_{3}=\alpha_{\mathrm{b} 1} \xi_{0} /\left[2 \alpha_{\mathrm{in} 1}\left(\psi_{\mathrm{cam} 0}-\psi_{\mathrm{e} 0}\right)^{1 / 2}+\alpha_{\mathrm{b} 1} \xi_{0}\right] \\
& \mathrm{K}_{4}=\alpha_{\mathrm{b} 1}\left(\psi_{\mathrm{cam} 0}-\psi_{\mathrm{e} 0}\right)^{1 / 2} /\left[\alpha_{\mathrm{in} 1}+0.5 \alpha_{\mathrm{b} 1} \xi_{0} /\left(\psi_{\mathrm{cam} 0}-\psi_{\mathrm{e} 0}\right)^{1 / 2}\right]
\end{aligned}
$$




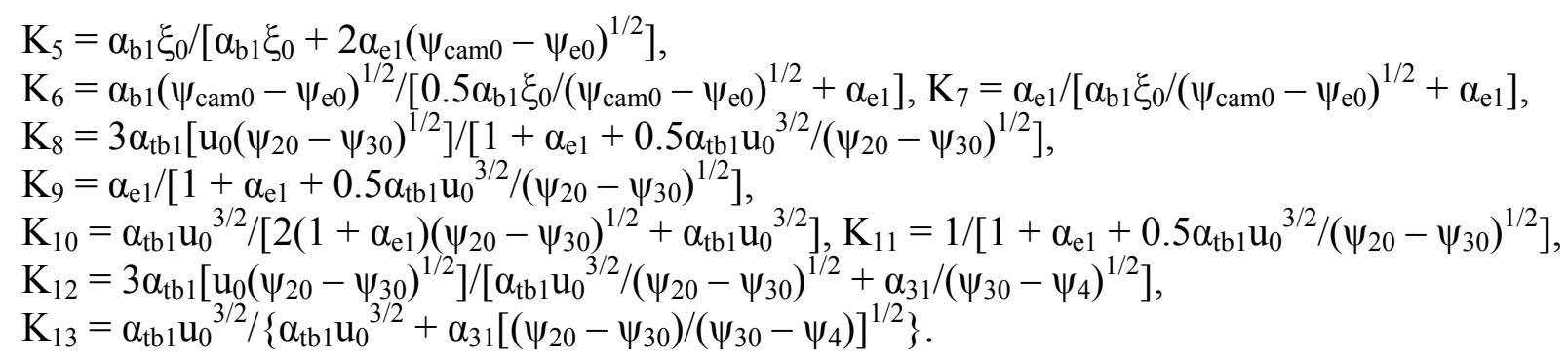

To investigate the frequency characteristics introduced replacement $p=i \omega$, where $i-$ imaginary unit, $\omega$ - angular frequency of the rotor The matrix $\mathrm{N}$ can be decomposed into real and imaginary parts: $\mathrm{N}(\mathrm{i} \omega)=\mathrm{N}_{\mathrm{R}}(\omega)+\mathrm{N}_{\mathrm{I}}(\omega)$. Real $\mathrm{W}_{\mathrm{R}}$ and imaginary $\mathrm{W}_{\mathrm{I}}$ parts of the vector of frequency transfer functions $\mathrm{W}(\mathrm{i} \omega)=\mathrm{N}^{-1} \mathrm{~B}=\mathrm{U}_{\mathrm{R}}+\mathrm{i} \omega \mathrm{U}_{\mathrm{I}}$ are $\mathrm{U}_{\mathrm{R}}=\left(\mathrm{N}_{\mathrm{R}} \mathrm{N}_{\mathrm{I}}^{-1} \mathrm{~N}_{\mathrm{R}}+\omega^{2} \mathrm{~N}_{\mathrm{I}}\right)^{-1} \mathrm{~N}_{\mathrm{R}} \mathrm{N}_{\mathrm{I}}^{-1} \mathrm{~B}, \mathrm{U}_{\mathrm{I}}=-\left(\mathrm{N}_{\mathrm{R}} \mathrm{N}_{\mathrm{I}}^{-1} \mathrm{~N}_{\mathrm{R}}+\right.$ $\left.+\omega^{2} \mathrm{~N}_{\mathrm{I}}\right)^{-1} \mathrm{~B}$. Amplitude frequency characteristic $\mathrm{A}_{\mathrm{u}}(\omega)=|\mathrm{W}(\mathrm{i} \omega)|=\left[\mathrm{W}_{\mathrm{R}}^{2}(\omega)+\mathrm{W}_{\mathrm{I}}^{2}(\omega)\right]^{1 / 2} \delta \psi_{1 \mathrm{a}} \mathrm{z}_{\mathrm{b}}$, where $\delta \psi_{1 \mathrm{a}}$ is amplitude of the dimensionless axial force variation.

To ensure dynamic stability of the system it is necessary to all real parts of roots of characteristic equation $|\mathrm{N}(\lambda)|=\mathrm{a}_{0} \lambda^{8}+\mathrm{a}_{1} \lambda^{7}+\ldots+\mathrm{a}_{8}=0$ were negative.

\section{Numerical Calculations}

Numerical calculations are carried out for the feed pump PE 600-300. Initial data for static calculations are nominal flow rate $\mathrm{Q}_{\mathrm{n}}=0.167 \mathrm{~m}^{3} / \mathrm{s}$, discharge pressure $\mathrm{p}_{\mathrm{b}}=32.3 \mathrm{MPa}$, angular rotor velocity $\omega=660 \mathrm{rad} / \mathrm{s}$, number of the pump stages $\mathrm{j}=7$; radiuses of impeller $\mathrm{R}_{0}=0.059 \mathrm{~m}$, $\mathrm{R}_{1}=0.097 \mathrm{~m}, \mathrm{R}_{2}=0.155 \mathrm{~m}$; axial force $\mathrm{T}_{\mathrm{n}}=330 \mathrm{kN}$. The base area $\mathrm{s}_{\mathrm{b}}=\mathrm{T}_{\mathrm{n}} / \mathrm{p}_{\mathrm{b}}=0.01 \mathrm{~m}^{2}$. Proportional coefficient $\mathrm{b}=1-\rho \omega^{2} \mathrm{R}_{2}{ }^{2} \mathrm{j}\left[1-\left(\mathrm{R}_{0}{ }^{2}+\mathrm{R}_{1}{ }^{2}\right) /\left(2 \mathrm{R}_{2}{ }^{2}\right)\right] /\left(8 \mathrm{p}_{\mathrm{b}}\right)=0.8$. The closing fluid parameters are density $\rho=1000 \mathrm{~kg} / \mathrm{m}^{3}$, dynamic viscosity $\mu=0.001$.

Inlet axial gap parameters are radius $\mathrm{r}_{\mathrm{c} 1}=0.072 \mathrm{~m}$, length $\mathrm{L}_{\mathrm{c} 1}=0.19 \mathrm{~m}$, gap $\mathrm{h}_{\mathrm{c} 1}=0.35 \mathrm{~mm}$; conductivity coefficient $\mathrm{g}_{1}=\pi \mathrm{r}_{\mathrm{c} 1} \mathrm{~h}_{\mathrm{c} 1}{ }^{3} /\left(6 \mu \mathrm{L}_{\mathrm{c} 1}\right)=8.6 \cdot 10^{-9} \mathrm{~m}^{3} /(\mathrm{Pa} \cdot \mathrm{s})$. Outlet axial gap parameters are radius $r_{c 3}=0.07 \mathrm{~m}$, length $L_{c 3}=0.26 \mathrm{~m}$, gap $h_{c 3}=0.35 \mathrm{~mm}$; sectional area of the liquid layer $\mathrm{f}_{\mathrm{c} 3}=$ $2 \pi \mathrm{r}_{\mathrm{c} 3} \mathrm{~h}_{\mathrm{c} 3}=1.54 \cdot 10^{-4} \mathrm{~m}^{2}$. External pressure $\mathrm{p}_{4}=0$. Estimated values of leakages $\mathrm{Q}=0.05 \mathrm{Q}_{\mathrm{n}}=$ $8.35 \cdot 10^{-3} \mathrm{~m}^{3} / \mathrm{s}$ and Reynolds number $\operatorname{Re}_{3}=\rho \mathrm{Q} /\left(\pi \mu \mathrm{r}_{\mathrm{c} 3}\right)=3.8 \cdot 10^{4}$; coefficient of hydraulic losses $\zeta_{3}=$ $\left.1.5+0.03 \mathrm{~L}_{\mathrm{c} 3} / \mathrm{h}_{\mathrm{c} 3}=23.8\right)$; conductivity coefficient $\mathrm{g}_{3}=\mathrm{f}_{\mathrm{c} 3} /\left(0.5 \rho \zeta_{3}\right)^{1 / 2}=1.4 \cdot 10^{-6} \mathrm{~m}^{3} /\left(\mathrm{Pa}^{1 / 2} \cdot \mathrm{s}\right)$.

Face gap parameters are inner and external radiuses $r_{2}=0.08 \mathrm{~m}, \mathrm{r}_{3}=0.12 \mathrm{~m}$; base value of the face gap $\mathrm{z}_{\mathrm{b}}=0.15 \mathrm{~mm}$; average sectional area of the liquid layer $\mathrm{f}_{\mathrm{tb}}=\pi\left(\mathrm{r}_{2}+\mathrm{r}_{3}\right) \mathrm{z}_{\mathrm{b}}=9.43 \cdot 10^{-5} \mathrm{~m}^{2}$; area $\mathrm{s}_{2}=\pi\left(\mathrm{r}_{2}{ }^{2}-\mathrm{r}_{\mathrm{c} 1}{ }^{2}\right)=3.59 \cdot 10^{-3} \mathrm{~m}^{2}$; face and effective areas $\mathrm{s}_{\mathrm{t}}=\pi\left(\mathrm{r}_{3}{ }^{2}-\mathrm{r}_{2}{ }^{2}\right)=0.025 \mathrm{~m}^{2}, \mathrm{~s}_{\mathrm{e}}=\mathrm{s}_{2}+$ $+0.5 \mathrm{~s}_{\mathrm{t}}=0.016 \mathrm{~m}^{2}$. Reynolds number $\mathrm{Re}_{\mathrm{tb}}=2 \rho \mathrm{Q} /\left[\pi \mu\left(\mathrm{r}_{2}+\mathrm{r}_{3}\right)\right]=2.7 \cdot 10^{4}$; coefficient of hydraulic losses $\zeta_{\mathrm{t}}=0.03\left(\mathrm{r}_{3}-\mathrm{r}_{2}\right) / \mathrm{z}_{\mathrm{b}}=8$; conductivity coefficient $\mathrm{g}_{\mathrm{tb}}=\mathrm{f}_{\mathrm{tb}} /\left(0.5 \rho \zeta_{\mathrm{tb}}\right)^{1 / 2}=1.49 \cdot 10^{-6} \mathrm{~m}^{3} /\left(\mathrm{Pa}^{1 / 2} \cdot \mathrm{s}\right)$.

Numerical calculations are carried out for the RPD parameters: inlet pressure $p_{\text {in }}=40 \mathrm{MPa}$, branch pipe length $\mathrm{L}_{\text {in }}=0.11 \mathrm{~m}$, inner diameter of the throttle orifice $\mathrm{d}_{\text {in }}=6\left(\mathrm{Q}^{2} / \mathrm{p}_{\mathrm{in}}\right)^{1 / 4}=0.006 \mathrm{~m}$; conductivity coefficient $\mathrm{g}_{\mathrm{in}}=\pi \mathrm{d}_{\mathrm{in}}{ }^{4} /\left(192 \mu \mathrm{L}_{\mathrm{in}}\right)=1.9 \cdot 10^{-7} \mathrm{~m}^{3} /(\mathrm{Pa} \cdot \mathrm{s})$. Average diameter of the saddle contact area $\mathrm{d}_{\mathrm{c}}=0.03 \mathrm{~m}$, base value of the throttling gap $\mathrm{x}_{\mathrm{b}}=1 \mathrm{~mm}$, leakage coefficient $\varphi_{\mathrm{c}}=0.54$; conductivity coefficient $\mathrm{g}_{\mathrm{b}}=\varphi_{\mathrm{c}} \pi \mathrm{d}_{\mathrm{c}} \mathrm{x}_{\mathrm{b}}(2 / \rho)^{1 / 2}=2.3 \cdot 10^{-6} \mathrm{~m}^{3} /\left(\mathrm{Pa}^{1 / 2} \cdot \mathrm{s}\right)$. Diameter and length of the fluid supplying throttle $\mathrm{d}_{\mathrm{e}}=3 \mathrm{~mm}, \mathrm{~L}_{\mathrm{e}}=0.075 \mathrm{~m}$; conductivity coefficient $\mathrm{g}_{\mathrm{e}}=\pi \mathrm{d}_{\mathrm{e}}{ }^{4} /\left(192 \mu \mathrm{L}_{\mathrm{e}}\right)=$ $=1.8 \cdot 10^{-8} \mathrm{~m}^{3} /(\mathrm{Pa} \cdot \mathrm{s})$. Spring force $\mathrm{F}_{\mathrm{spr}}=0.01 \mathrm{~T}_{\mathrm{n}}=3.3 \mathrm{kN}$. Dimensionless parameters $\psi_{\mathrm{in}}=\mathrm{p}_{\mathrm{in}} / \mathrm{p}_{\mathrm{b}}=$ $1.24, \sigma=\mathrm{s}_{\mathrm{e}} / \mathrm{s}_{\mathrm{b}}=1.6, \chi=\mathrm{F}_{\mathrm{spr}} /\left(\mathrm{p}_{\mathrm{b}} \mathrm{s}_{\mathrm{b}}\right)=0.01, \psi_{4}=\mathrm{p}_{4} / \mathrm{p}_{\mathrm{b}}=0 ; \alpha_{\mathrm{in} 1}=\mathrm{g}_{\mathrm{in}} / \mathrm{g}_{1}=22.5, \alpha_{\mathrm{b} 1}=\mathrm{g}_{\mathrm{b}} /\left(\mathrm{g}_{1} \mathrm{p}_{\mathrm{b}}{ }^{1 / 2}\right)=$ $0.05, \alpha_{\mathrm{e} 1}=\mathrm{g}_{\mathrm{e}} / \mathrm{g}_{1}=2.1, \alpha_{\mathrm{tb} 1}=\mathrm{g}_{\mathrm{tb}} /\left(\mathrm{g}_{1} \mathrm{p}_{\mathrm{b}}{ }^{1 / 2}\right)=0.03, \alpha_{31}=\mathrm{g}_{3} /\left(\mathrm{g}_{1} \mathrm{p}_{\mathrm{b}}{ }^{1 / 2}\right)=0.03$. The minimum pressure difference $\delta \psi_{\text {min }}=\alpha_{31}\left(1+\alpha_{\mathrm{e} 1}\right) / \alpha_{\mathrm{e} 1}[1-(\mathrm{b}-\chi) / \sigma]^{1 / 2}=0.03 ; \delta \psi=0.04$. The critical value of the discharge pressure $\psi_{1}{ }^{\mathrm{cr}}=\left\{\left[\alpha_{\mathrm{e} 1} / \alpha_{31} \cdot \delta \psi /\left(1+\alpha_{\mathrm{e} 1}\right)\right]^{2}-\delta \psi \cdot \alpha_{\mathrm{e} 1} /\left(1+\alpha_{\mathrm{e} 1}\right)+\chi / \sigma\right\} /(1-\mathrm{b} / \sigma)=1.67$. The closing fluid flow rate $\mathrm{Q}_{\mathrm{e}}=\mathrm{g}_{\mathrm{e}} \mathrm{p}_{\mathrm{b}} \delta \psi /\left(1+\alpha_{\mathrm{e} 1}\right)=0.007 \mathrm{~m}^{3} / \mathrm{s}$.

Static characteristics are represented on Fig. 3. 


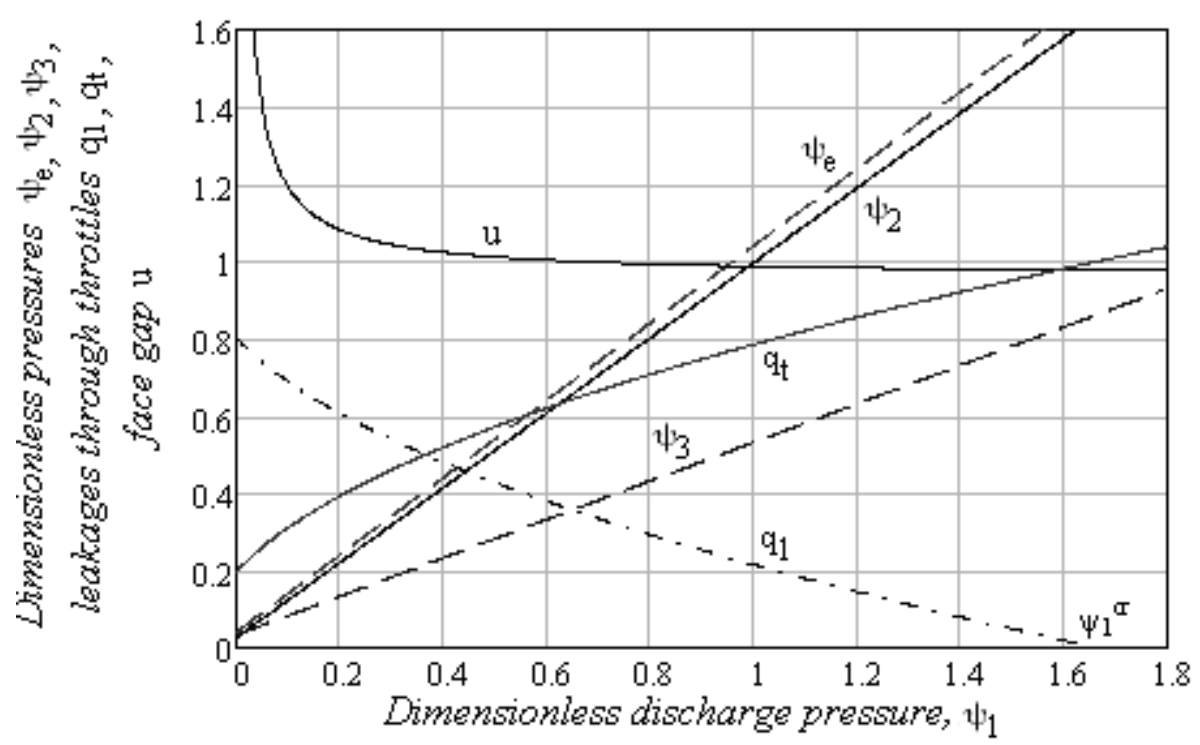

Fig. 3. Static characteristics: $\psi_{\mathrm{e}, 2,3}-$ dimensionless pressures in the chambers of the hydraulic path; $\mathrm{u}$ - dimensionless face gap; $\mathrm{q}_{1, \mathrm{t}}-$ dimensionless leakages through throttles

Initial data for dynamic analysis are parameters of the static calculations: $\mathrm{u}_{0}=1, \xi_{0}=1.3$, $\psi_{\mathrm{cam} 0}=1.2, \psi_{\mathrm{e} 0}=1.04, \psi_{20}=1.03, \psi_{30}=0.53$. Physical and geometrical parameters: rotor and rod masses $\mathrm{m}_{\mathrm{r}}=3$ tons, $\mathrm{m}_{0}=2 \mathrm{~kg}$; inner and external membrane diameters $\mathrm{d}_{\mathrm{m} 1}=0.05 \mathrm{~m}, \mathrm{~d}_{\mathrm{m} 2}=0.15 \mathrm{~m}$; membrane area $\mathrm{s}_{\mathrm{m}}=\pi\left(\mathrm{d}_{\mathrm{m} 2}{ }^{2}-\mathrm{d}_{\mathrm{m} 1}{ }^{2}\right) / 4=0.016 \mathrm{~m}^{2}$; dimensionless area $\sigma_{\mathrm{m}}=\mathrm{s}_{\mathrm{m}} / \mathrm{s}_{\mathrm{b}}=1.54$; contact area of the RPD saddle $\mathrm{s}_{\mathrm{c}}=\pi \mathrm{d}_{\mathrm{c}}{ }^{2} / 4=7 \cdot 10^{-4} \mathrm{~m}^{2}$; stiffness coefficients $\mathrm{k}_{\mathrm{spr}}=1 \cdot 10^{8} \mathrm{~N} / \mathrm{m}, \mathrm{k}_{\mathrm{reg}}=1 \cdot 10^{7} \mathrm{~N} / \mathrm{m}$; modulus of elasticity of closing fluid $\mathrm{E}=2.2 \cdot 10^{9} \mathrm{~N} / \mathrm{m}^{2}$; chambers' volumes $\mathrm{V}_{\text {cam }}=5.3 \cdot 10^{-7} \mathrm{~m}^{3}$, $\mathrm{V}_{\mathrm{m}}=1.4 \cdot 10^{-5} \mathrm{~m}^{3}, \mathrm{~V}_{2}=4.7 \cdot 10^{-5} \mathrm{~m}^{3}, \mathrm{~V}_{3}=1 \cdot 10^{-4} \mathrm{~m}^{3}$. Amplitude of axial force variation $\delta \psi_{1 \mathrm{a}}=0.2$.

Time constants, damping coefficients and amplification factors are $\mathrm{T}_{1}=5.5 \mathrm{~ms}, \mathrm{~T}_{2}=0.45 \mathrm{~ms}$, $\mathrm{T}_{3}=1.2 \cdot 10^{-9} \mathrm{~s}, \mathrm{~T}_{4}=0.02 \mathrm{~ms}, \mathrm{~T}_{5}=3.5 \cdot 10^{-7} \mathrm{~s}, \mathrm{~T}_{6}=0.4 \mathrm{~ms}, \mathrm{~T}_{7}=8.1 \cdot 10^{-7} \mathrm{~s}, \mathrm{~T}_{8}=0.3 \mathrm{~ms}, \mathrm{~T}_{9}=0.1$ $\mathrm{ms} ; \tau_{3}=4.1 \cdot 10^{-7} \mathrm{~s}, \tau_{5}=3.6 \cdot 10^{-7} \mathrm{~s}, \tau_{6}=2.8 \cdot 10^{-6} \mathrm{~s}, \tau_{7}=0.1 \mathrm{~ms} ; \zeta_{1}=\zeta_{2}=0.05 ; \mathrm{K}_{1}=0.01, \mathrm{~K}_{2}=$ 0.061 ,

$\mathrm{K}_{3}=0.003, \mathrm{~K}_{4}=0.001, \mathrm{~K}_{5}=0.03, \mathrm{~K}_{6}=0.01, \mathrm{~K}_{7}=0.94, \mathrm{~K}_{8}=0.02, \mathrm{~K}_{9}=0.67, \mathrm{~K}_{10}=0.007$, $\mathrm{K}_{11}=0.32, \mathrm{~K}_{12}=0.78, \mathrm{~K}_{13}=0.52$.

Amplitude-frequency characteristic is represented on Fig. 4. The first and second resonance frequencies of axial oscillations of the rotor are $\omega_{1}=1920 \mathrm{rad} / \mathrm{s}$ and $\omega_{2}=2907 \mathrm{rad} / \mathrm{s}$. Resonance amplitudes of axial oscillations of the pump rotor are $A_{1}=29 \mathrm{um}$ and $A_{2}=17 \mathrm{~mm}$. Amplitude $\mathrm{A}_{0}=8 \mathrm{um}$ for the operating mode $\omega_{0}=660 \mathrm{rad} / \mathrm{s}$ corresponds to accident-free regime.

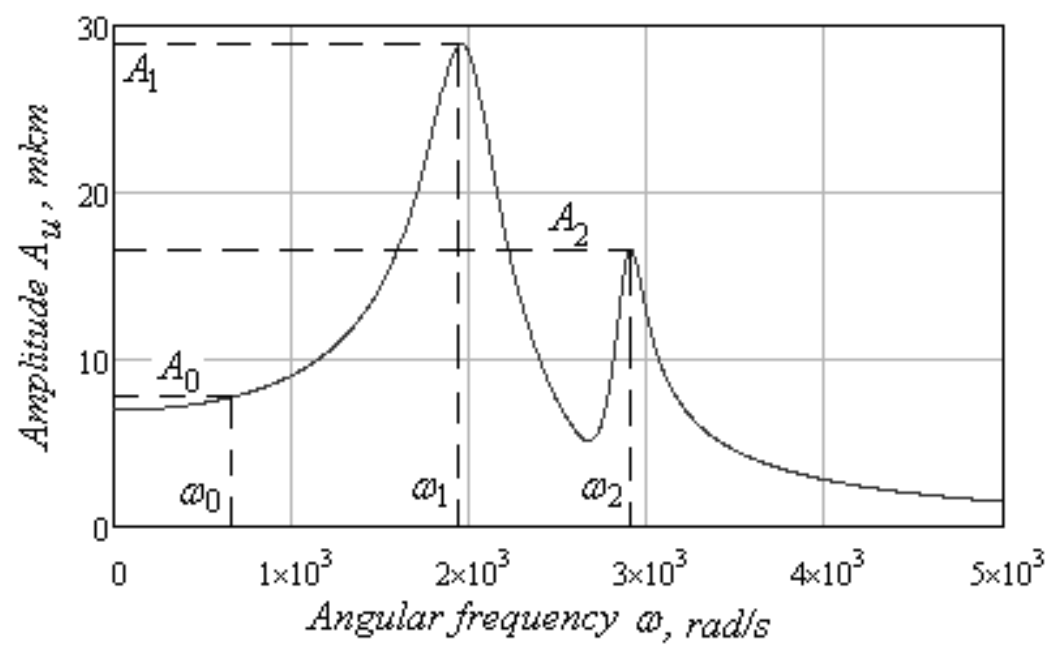

Fig. 4. Amplitude-frequency characteristic 
Coefficients of characteristic polynomial $\mathrm{a}_{0}=0.046, \mathrm{a}_{1}=3.1 \cdot 10^{-5}, \mathrm{a}_{2}=1.1 \cdot 10^{-8}, \mathrm{a}_{3}=1.4 \cdot 10^{-11}$, $\mathrm{a}_{4}=5.3 \cdot 10^{-16}, \mathrm{a}_{5}=1.2 \cdot 10^{-18}, \mathrm{a}_{6}=6.2 \cdot 10^{-23}, \mathrm{a}_{7}=3.7 \cdot 10^{-28}, \mathrm{a}_{8}=1.4 \cdot 10^{-34}$ are all positive, and roots of characteristic equation $\lambda_{1,2}=-231.2 \pm 1920 \mathrm{i}, \lambda_{3,4}=-430.1 \pm 2907 \mathrm{i}, \lambda_{5}=-1.2 \cdot 10^{3}, \lambda_{6}=-2.2 \cdot 10^{4}$, $\lambda_{6}=-1.6 \cdot 10^{5}, \lambda_{6}=-2.5 \cdot 10^{6}$ have negative real parts, that testifies to the stability of the dynamic system. Moreover, two pairs of complex roots include imaginary components that are equal to resonance frequencies $\omega_{1}=1920 \mathrm{rad} / \mathrm{s}, \omega_{2}=2907 \mathrm{rad} / \mathrm{s}$ (Fig. 4).

\section{Summary}

The closing rotor balancing device of the multistage centrifugal pump with regulator of pressure difference acts as the end seal and the hydrostatic bearing with self-regulating gap and fluid leakages. The main advantages of this design are absence of end seals and bearings and leakage of working fluid.

As an example for feed pump PE 600-300 were stated static characteristics: pressures in chambers of the hydraulic path, face gap and leakages through available throttles. The minimum pressure difference, critical value of the discharge pressure and closing fluid flow rate are stated.

Amplitude frequency characteristic and dynamic stability of the system are investigated. The lowest resonance frequency is 2.9 times more the operating frequency.

At this stage dynamic stability of the system is implemented by numerical verification by linearized equations. In perspective it is necessary to define conditions and boundaries for dynamic stability by Hurwitz criterion and investigate mathematical model without linearization on the basis of numerical methods for solving nonlinear systems of differential higher order equations.

\section{References}

[1] A. Korczak, W. Marcinkowski, G. Peczkis, Zespół tarczy odciążającej siłę osiową w wirnikowej sprężarce promieniowej (2004), Urząd Patentowy Rzeczpospolitej Polskiej, Patent Nr 207968 - 04.03.2011.

[2] A. Korczak, G. Peczkis, W. Marcinkowski, Using the locking hydraulic device for rotor balancing, Bulletin of Sumy State University, Series Engineering, 1 (2005) 68-76.

[3] I. Pavlenko, Static analysis of the locking automatic balancing device of the centrifugal pumps, $12^{\text {th }}$ International Scientific and Engineering Conference Hermetic sealing, vibration reliability and ecological safety of pump and compressor machinery, Kielce - Przemyśl, 2 (2008) 165 - 172.

[4] I. Pavlenko, Dynamic analysis of the locking automatic balancing device of the centrifugal pump, Journal of mechanical engineering "Strojnícky časopis", Bratislava, Institute of Materials and Machine Mechanics, Slovak Academy of Science, 2 (60) (2009) 75 - 86.

[5] W. Marcinkowski, A. Zagorulko, S. Mishchenko, Dynamics of the locking automatic balancing device, Bulletin of Sumy State University, Series Engineering, 2 (2010) 24-34.

[6] I. Pavlenko, Investigation of nonlinear rotor oscillations of the multistage centrifugal compressor with the automatic balancing device, Journal of Manufacturing Engineering, Prešov, Slovak Republic, 12 (3-4) (2013) 35-39. 